\title{
Comment on "The impact of visceral fat and levels of vitamin D on coronary artery calcification"
}

\author{
Juan Zhang' ${ }^{1}$, Lianping $\mathrm{He}^{1 *}$
}

Dear Editor,

It is our pleasure to read this interesting study by Isa Galvão Rodrigues et al. ${ }^{1}$ This study shows that excess visceral fat was associated with subclinical atherosclerosis, regardless of other risk factors for cardiovascular disease. Furthermore, serum levels of 25-hydroxyvitamin D were not associated with calcification of coronary arteries (CCA) in its early stages. Although the study plays a vital role in the assessment of coronary atherosclerosis, I believe that there are some issues that should be discussed further.

First of all, in the results section, it is mentioned that hypertension and age $>60$ years were associated with CCA after adjusting for confounding variables such as gender, presence of diabetes, subcutaneous adipose tissue (SAT), and body mass index (BMI) (Table 3). However, the table fails to provide detailed parameters such as $\mathrm{OR}$ value and $\mathrm{p}$-value of the items that eliminate confounding factors.

Furthermore, it is a vague statement that this study puts forward the point of relatively small sample size in the discussion section. The study does not calculate the sample size and degree of certainty, so this statement is inappropriate.

\section{AUTHORS" CONTRIBUTION}

THL, LPH: Conceptualization, Data curation, Formal analysis, Writing - original draft, Writing - review\& editing.

\section{REFERENCE}

1. Rodrigues IG, Pinho CPS, Sobral Filho D, Leão APD, Oliveira MCM, Barbosa GP et al. The impact of visceral fat and levels of vitamin D on coronary artery calcification. Rev Assoc Med Bras. 2021;67(1):88-93. https://doi.org/10.1590/1806-9282.67.01.20200388

${ }^{1}$ Taizhou University, School of Medicine - Zhejiang, China.

*Corresponding author: lianpinghe@tzc.edu.cn

Conflicts of interest: the authors declare there are no conflicts of interest. Funding: none.

Received on July 15, 2021. Accepted on July 25, 2021. 